\title{
Generalized gene co-expression analysis via subspace clustering using low-rank representation
}

\author{
Tongxin Wang ${ }^{1}$, Jie Zhang ${ }^{2}$ and Kun Huang ${ }^{3,4^{*}}$ \\ From The 12th International Conference on Computational Systems Biology (ISB 2018) \\ Guiyang, China. 18-21 August 2018
}

\begin{abstract}
Background: Gene Co-expression Network Analysis (GCNA) helps identify gene modules with potential biological functions and has become a popular method in bioinformatics and biomedical research. However, most current GCNA algorithms use correlation to build gene co-expression networks and identify modules with highly correlated genes. There is a need to look beyond correlation and identify gene modules using other similarity measures for finding novel biologically meaningful modules.

Results: We propose a new generalized gene co-expression analysis algorithm via subspace clustering that can identify biologically meaningful gene co-expression modules with genes that are not all highly correlated. We use low-rank representation to construct gene co-expression networks and local maximal quasi-clique merger to identify gene co-expression modules. We applied our method on three large microarray datasets and a single-cell RNA sequencing dataset. We demonstrate that our method can identify gene modules with different biological functions than current GCNA methods and find gene modules with prognostic values.

Conclusions: The presented method takes advantage of subspace clustering to generate gene co-expression networks rather than using correlation as the similarity measure between genes. Our generalized GCNA method can provide new insights from gene expression datasets and serve as a complement to current GCNA algorithms.
\end{abstract}

Keywords: Gene co-expression network analysis, Subspace clustering, Low-rank representation

\section{Background}

Gene Co-expression Network Analysis (GCNA) is a popular method in bioinformatics and biomedical research to construct gene co-expression networks and detect coexpressed genes. It has been widely utilized in many applications, such as gene function prediction [1-3], disease biomarker discovery $[4,5]$, protein-protein interaction (PPI) inference [6] and genetic variants detection in cancers $[7,8]$.

\footnotetext{
*Correspondence: kunhuang@iu.edu

${ }^{3}$ Department of Medicine, Indiana University School of Medicine, 46202

Indianapolis, IN, USA

${ }^{4}$ Regenstrief Institute, 46202 Indianapolis, IN, USA

Full list of author information is available at the end of the article
}

Many GCNA algorithms have been developed to identify modules of co-expressed genes [3, 9-13]. One of the widely used GCNA tools is the WGCNA package developed by Horvath's group [10], which finds modules of highly correlated genes in weighted gene co-expression networks. Local maximal Quasi-Clique Merger (lmQCM) [13] is another weighted GCNA algorithm that allows overlap between gene modules, which is consistent with the fact that the same gene may participate in multiple biological processes.

Mathematically, given the expression matrix $\mathbf{G}$ for $K$ genes and $N$ samples

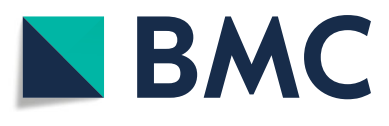

(c) The Author(s). 2019 Open Access This article is distributed under the terms of the Creative Commons Attribution 4.0 International License (http://creativecommons.org/licenses/by/4.0/), which permits unrestricted use, distribution, and reproduction in any medium, provided you give appropriate credit to the original author(s) and the source, provide a link to the Creative Commons license, and indicate if changes were made. The Creative Commons Public Domain Dedication waiver (http://creativecommons.org/publicdomain/zero/1.0/) applies to the data made available in this article, unless otherwise stated. 


$$
\mathbf{G}=\left[\begin{array}{c}
\mathbf{g}_{\mathbf{1}} \\
\mathbf{g}_{\mathbf{2}} \\
\cdots \\
\mathbf{g}_{\mathbf{K}}
\end{array}\right] \in \Re^{K \times N}
$$

with $\mathbf{g}_{\mathbf{i}}{ }^{T} \in \mathfrak{R}^{N}$ being the expression profile for gene $i$ $(i \in\{1,2, \ldots, K\})$, the geneco-expression network can be represented by a matrix $\mathbf{W} \in \Re^{K \times K}$, where entry $W_{i, j}$ represents the co-expression similarity between expression profiles of a pair of genes $\mathbf{g}_{\mathbf{i}}$ and $\mathbf{g}_{\mathbf{j}}(i, j \in\{1,2, \ldots, K\})$. Commonly, this similarity is measured using correlation coefficients, with Pearson Correlation Coefficient (PCC) or Spearman Correlation Coefficient (SCC) being the most widely used ones $[3,9,13]$. When using PCC to measure co-expression similarity, we obtain gene modules with linearly correlated gene expression profiles. In this case, expression profiles of gene $i$ and $j(i \neq j)$ from the same co-expression module differ by a scale and a shift, i.e.,

$$
\mathbf{g}_{\mathbf{i}}=\alpha_{j} \mathbf{g}_{\mathbf{j}}+\beta_{j} \cdot \mathbf{1}_{1 \times N}
$$

where $\alpha_{j}$ and $\beta_{j}$ are scalars. It can be easily shown that the rank of such expression matrix for a co-expression module $\mathbf{G}_{\mathbf{c}}$ is 2. In other words, expression profiles for genes in a co-expression module can be approximated as a subspace with dimensionality of two in a $N$-dimensional space. In addition, if we designate

$$
\overline{\mathbf{g}_{\mathbf{i}}}=\mathbf{g}_{\mathbf{i}}-\bar{g}_{i} \cdot \mathbf{1}_{1 \times N}
$$

as the centralized version of $\mathbf{g}_{\mathbf{i}}$, where $\bar{g}_{i}$ is the mean of entries in $\mathbf{g}_{\mathbf{i}}$, then $\overline{\mathbf{g}}_{\mathbf{i}} \mathbf{s}$ from the same co-expression module can be approximated by a 1-dimensional subspace.

However, apart from grouping genes that are linearly correlated into modules, simple relationships such as

$$
\mathbf{g}_{\mathbf{i}}=\alpha_{j} \mathbf{g}_{\mathbf{j}}+\alpha_{k} \mathbf{g}_{\mathbf{k}}+\alpha_{m} \mathbf{g}_{\mathbf{m}}
$$

where $\mathbf{g}_{\mathbf{j}}, \mathbf{g}_{\mathbf{k}}$ and $\mathbf{g}_{\mathbf{m}}$ are linearly independent and $\alpha_{j}, \alpha_{k}, \alpha_{m} \neq 0$, cannot be captured using the traditional PCC based co-expression formulation. In this case, expression profiles for gene $i, j, k$ and $m$ cannot be approximated using a 2-dimensional subspace (in this example, they form a 3-dimensional subspace). However, it can be conceived that in biology, such coordinated gene activities may play important roles in complex processes and pathways. Therefore, there is a need to generalize the co-expression formulation to accommodate relationships between genes beyond pairwise relationships.

Discovering gene modules with such coherent relationships implies detecting low-dimensional subspaces in a higher dimensional space. Subspace clustering [14] is a research field in signal processing and machine learning for such purpose. The goal of subspace clustering is separating data according to their underlying subspaces, which could have different dimensionalities that are larger than one. Subspace clustering has found numerous applications in image processing and computer vision [15-18], as well as in bioinformatics [19-21].

One of the popular approaches for subspace clustering is Sparse Subspace Clustering (SSC) [22]. SSC is based on the affinity matrix defined by the sparsest representation produced by $l_{1}$-minimization and subspace segmentation using spectral clustering. However, SSC may not be able to capture the global structure of the data accurately, which could affect the performance of the algorithm when data is highly corrupted. However, large biomedical datasets often contain large amount of noise and outliers. In addition, spectral clustering assigns every data point to a certain cluster, which can potentially bias the clustering structure.

Therefore, in this paper, we propose to use Low-Rank Representation (LRR) approach to construct gene coexpression networks from high-throughput gene expression data and use ImQCM to further group genes from the same subspace into gene modules. The ImQCM algorithm is developed by us as an extension of the QCM algorithm. It is a greedy algorithm for identifying highly connected modules in a large network with high efficiency. In addition, it allows overlap between clusters, which fits well with the notion that genes can participate in different functions and pathways.

Comparing to traditional GCNA algorithms based on correlation, our method provides a generalized formulation and can be applied to identify gene modules with expression matrices of higher intrinsic dimensionalities. This will help to discover new biological relationships, functions and pathways. Our method can also serve as a complement to current GCNA algorithms. Moreover, since LRR finds the lowest rank representation of all data jointly [18] and the corruption of data will largely increase the rank, LRR is robust to noise and outliers, making it suitable for analyzing high-throughput gene expression data. The contribution of this paper is a generalized gene co-expression network mining approach that is based on subspace clustering and demonstration of the effectiveness of this approach using real biomedical data.

\section{Methods}

\section{Subspace clustering of gene expression data using LRR and ImQCM}

Consider a data matrix $\mathbf{X} \in \Re^{D \times N}$ (each column is a sample) where each sample can be represented by a linear combination of columns in a dictionary $\mathbf{A} \in \mathfrak{R}^{D \times M}$.

$$
\mathbf{X}=\mathbf{A Z}
$$

$\mathbf{Z} \in \mathfrak{R}^{M \times N}$ is a matrix with the $i$-th column being the representation of the $i$-th column in $\mathbf{X}$. Introduced by Liu et al., LRR [18] uses low rankness of a matrix to capture the structure of the data and looks for a representation $\mathbf{Z}$ of data $\mathbf{X}$ by solving the following problem. 


$$
\begin{array}{cl}
\min _{\mathbf{Z}} & \operatorname{rank}(\mathbf{Z}) \\
\text { s.t. } & \mathbf{X}=\mathbf{A Z}
\end{array}
$$

However, due to the discrete nature of the rank function, Problem (4) is difficult to solve. Instead, the following convex optimization problem is suggested as a surrogate for Problem (4) by matrix completion methods,

$$
\begin{array}{cl}
\min _{\mathbf{Z}} & \|\mathbf{Z}\|_{*} \\
\text { s.t. } & \mathbf{X}=\mathbf{A Z}
\end{array}
$$

where $\|\cdot\|_{*}$ is the nuclear norm of a matrix.

In the case of gene expression data, we represent a dataset with $K$ genes and $N$ samples using a matrix $\mathbf{G}$ of size $K \times N$. In order to cluster genes into their respective subspaces, we need to compute an affinity matrix encoding the pairwise similarities between genes by using the gene expression matrix itself as the dictionary [18]. We use $\mathbf{G}^{T}$ to substitute $\mathbf{X}$ in Problem (5) and Problem (5) becomes:

$$
\begin{array}{ll}
\min _{\mathbf{Z}} & \|\mathbf{Z}\|_{*} \\
\text { s.t. } & \mathbf{G}^{T}=\mathbf{G}^{T} \mathbf{Z}
\end{array}
$$

In reality, gene expression data often contains noise that could not be neglected. To take noise into account, we could modify the objective of Problem (6) into

$$
\begin{array}{cl}
\min _{\mathbf{Z}, \mathbf{E}} & \|\mathbf{Z}\|_{*}+\lambda\|\mathbf{E}\|_{2,1} \\
\text { s.t. } & \mathbf{G}^{T}=\mathbf{G}^{T} \mathbf{Z}+\mathbf{E}
\end{array}
$$

where $\lambda>0$ is used to balance the effect of low rankness and noise. Problem (7) could be solved by solving an Augmented Lagrange Multiplier (ALM) problem using inexact ALM algorithms. After solving Problem (7), we define the adjacency matrix $\mathbf{W}$ of a weighted, undirected graph between genes based on the "lowest-rank representation" $\mathbf{Z}^{*}$. The weight between gene $i$ and $j, W_{i j}$, is computed by $\left|Z_{i j}^{*}\right|+\left|Z_{j i}^{*}\right|$. As in [17], to deal with different norms of gene expression levels in $\mathbf{G}$ and ensure that the largest weights for all the genes are of the same scale, we also normalize the columns of $\mathbf{Z}^{*}$ as $\mathbf{z}_{\mathbf{i}}=\mathbf{z}_{\mathbf{i}} /\left\|\mathbf{z}_{\mathbf{i}}\right\|_{\infty}$, where $\mathbf{z}_{\mathbf{i}}$ is the $i$-th column of $\mathbf{Z}^{*}$.

Once we obtain the weighted network of genes, we need to cluster genes into their respective subspaces and perform further analysis on gene modules. Instead of using normalized cuts in [18] or spectral clustering in [17], we apply a recently developed weighted network mining algorithm called ImQCM [13]. Unlike normalized cuts or spectral clustering, which partitions genes into disjoint sets and does not allow overlaps between clusters, lmQCM is a greedy approach that allows genes to be shared among multiple clusters or not included in any cluster. This is consistent with subspace clustering problems where two subspace clusters can share some common genes or some genes may not belong to any subspace cluster [23]. Also, genes can participate in multiple biological processes, which could be represented by different clusters. Another advantage of ImQCM is that it can find gene co-expression modules potentially associated with Copy Number Variations (CNVs) in cancer development [13]. The lmQCM algorithm has four parameters $\gamma, \alpha, t$ and $\beta . \gamma$ determines if a new module can be initiated by setting the weight threshold for the first edge of the module, and has the largest influence on the results. We use the default setting of $\alpha=1, t=1, \beta=0.4$ in this paper and tune $\gamma$ empirically.

Our method combines the strength of LRR and ImQCM, which we outline in Algorithm 1.

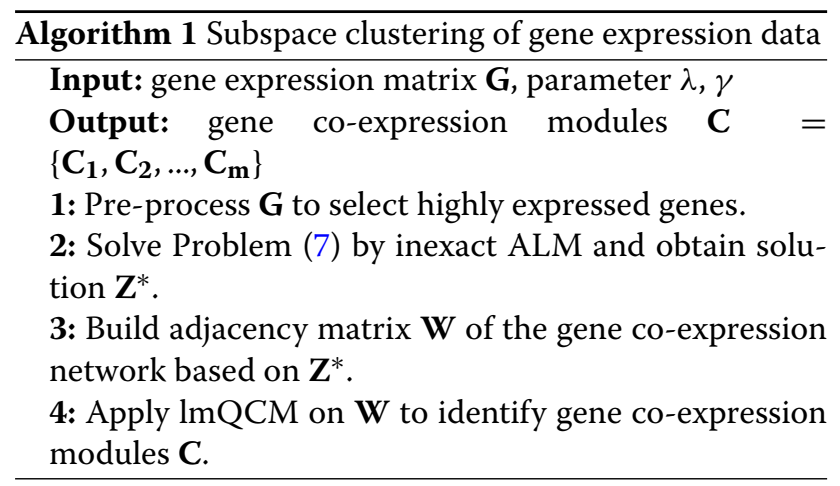

There are two parameters we need to choose in our method: $\lambda$ for LRR and $\gamma$ for lmQCM. In order to choose a set of parameters that is able to produce robust results, we randomly partition the dataset into 10 folds with the same size. Each time we use 9 folds to generate gene modules an $\mathrm{d}$ we can get 10 sets of gene modules. We use $g_{10}$ to denote the number of genes that appear in all 10 sets of gene modules and use $g_{1}$ to denote the number of genes that appear in at least one set of gene modules. The value of $g_{10} / g_{1}$ can indicate the stability of the results under a certain set of parameters. We do not consider the parameters that can only identify less than $\mathrm{nMdl}_{t h r}$ modules and choose the parameters that can produce the highest $g_{10} / g_{1}$ value.

\section{Functional enrichment analysis}

Gene set functional enrichment analysis is a method to find biological annotations that are significant in a set of genes. In this study, we use annotations of Gene Ontology Biological Process (GO BP) terms from Gene Ontology Consortium and annotations of chromosome bands of protein coding genes from HGNC database. Hypergeometric probability density function from MATLAB Statistics and Machine Learning Toolbox with false discovery rate correction from MATLAB Bioinformatics Toolbox is used to determine the statistical significance. To provide 
meaningful results, we only perform functional enrichment analysis on gene modules with at least 10 genes and at most 500 genes.

For chromosome regions enriched by the identified gene modules, we are interested in if patients in other cohorts with the same disease have CNVs in these regions. We use OncoPrint visualization from the cBioPortal [24] website to investigate the Copy Number Alternations (CNAs) in selected genes. The Cancer Genome Atlas (TCGA) bladder cancer dataset (406 patients) was used to validate chromosome bands enrichment results of GSE31684. TCGA breast cancer dataset (996 patients) and METABRIC [25] breast cancer dataset (2051 patients) were used to validate chromosome bands enrichment results of GSE54002 and GSE102484.

\section{Gene expression data}

Three large gene expression datasets were obtained from the NCBI Gene Expression Omnibus (GEO) and all datasets were generated using the Affymetrix Human Genome U133 Plus 2.0 Array Genechip with more than 54,000 probesets. Details of these datasets are summarized in Table 1.

To reduce the size of affinity matrix between genes, preprocessing of each dataset is performed to select highly expressed genes. Firstly, only probesets with known associated genes are selected. If multiple probesets correspond to the same gene, only the probeset with the highest mean expression value is retained. Next, genes with low mean expression levels (bottom 20\%) and low variance (bottom $10 \%$ ) are removed using functions from MATLAB Bioinformatics Toolbox. Finally, we retain 10,000 genes with the highest mean expression levels for further analysis.

\section{Results}

\section{LRR finds gene modules with different structures}

Centralized Concordance Index (CCI) [26] is a linear algebraic based index for evaluating the concordance of gene co-expression modules from GCNA. A high CCI value suggests genes in a gene module are highly correlated, while a low CCI value suggests higher intrinsic dimensionality of expression profiles in a module. For example, when PCC between each pair of genes in a module is $1, \mathrm{CCI}$ of this module is 1 .

To compare our method with current correlation based GCNA algorithms, besides LRR, we also used PCC to generate the adjacency matrix of a gene co-expression

Table 1 Summary of microarray datasets

\begin{tabular}{llll}
\hline Dataset & Disease & Number of samples & Platform \\
\hline GSE31684 & bladder cancer & 93 & GPL570 \\
GSE54002 & breast cancer & 433 & GPL570 \\
GSE102484 & breast cancer & 683 & GPL570 \\
\hline
\end{tabular}

network and applied ImQCM to identify gene coexpression modules. We calculated CCI of each gene module identified using PCC based method and our LRR based method. We observe that gene modules identified by LRR based method have significantly lower CCI values than those identified by PCC based method using Kolmogorov-Smirnov test (Table 2). This suggests that LRR, combined with ImQCM, can find gene coexpression modules from subspaces with higher dimensionality than current linear correlation based GCNA algorithms.

We also applied one of the state-of-the-art GCNA algorithms, WGCNA [10], for comparison. Since WGCNA partitions all the genes after pre-processing into disjoint sets rather than just identifies highly connected modules, CCI values of modules identified by WGCNA are not comparable with our ImQCM based results and we did not apply Kolmogorov-Smirnov tests on CCI values of identified gene modules between our method and WGCNA.

\section{Difference in $\mathrm{CCl}$ contributes to difference in enriched biological annotations}

In the previous section, we have shown that gene modules identified by LRR based method have different structures from those identified by PCC based method, indicated by lower $\mathrm{CCI}$ values. In this section, we further demonstrate that such difference could lead to different enrichment results in biological annotations, such as GO BPs and chromosome bands. This suggests that our method is able to provide different biological insights than current correlation based GCNA algorithms and serve as a complement to current methods.

We first compare three similar gene co-expression modules identified in GSE54002 dataset: $\mathrm{LRR}_{21}$ identified by our method, $\mathrm{PCC}_{30}$ identified by PCC based method and $\mathrm{WGCNA}_{44}$ identified by WGCNA. These three modules share a large fraction of the same genes and a Venn diagram of genes in these modules is shown in Fig. 1.

Figure 1 also shows heatmaps of gene expression profiles in these three modules. Expression levels of each gene have been standardized across different samples in

Table 2 Gene modules identified by LRR, PCC and WGCNA

\begin{tabular}{|c|c|c|c|}
\hline Dataset & GSE31684 & GSE54002 & GSE102484 \\
\hline $\mathrm{nMdl}_{L R R}$ & 26 & 76 & 63 \\
\hline$\overline{\mathrm{CCl}}_{\mathrm{LRR}}$ & 0.487 & 0.471 & 0.457 \\
\hline nMdlpcc & 97 & 100 & 81 \\
\hline$\overline{\mathrm{CCl}}_{\mathrm{PCC}}$ & 0.552 & 0.518 & 0.496 \\
\hline $\begin{array}{l}\text { Kolmogorov-Smirnov } \\
\text { test } P \text { value }\end{array}$ & $2.43 e-02$ & $1.09 \mathrm{e}-04$ & $9.22 \mathrm{e}-04$ \\
\hline $\mathrm{nMdl}_{\text {WGCNA }}$ & 56 & 147 & 81 \\
\hline
\end{tabular}




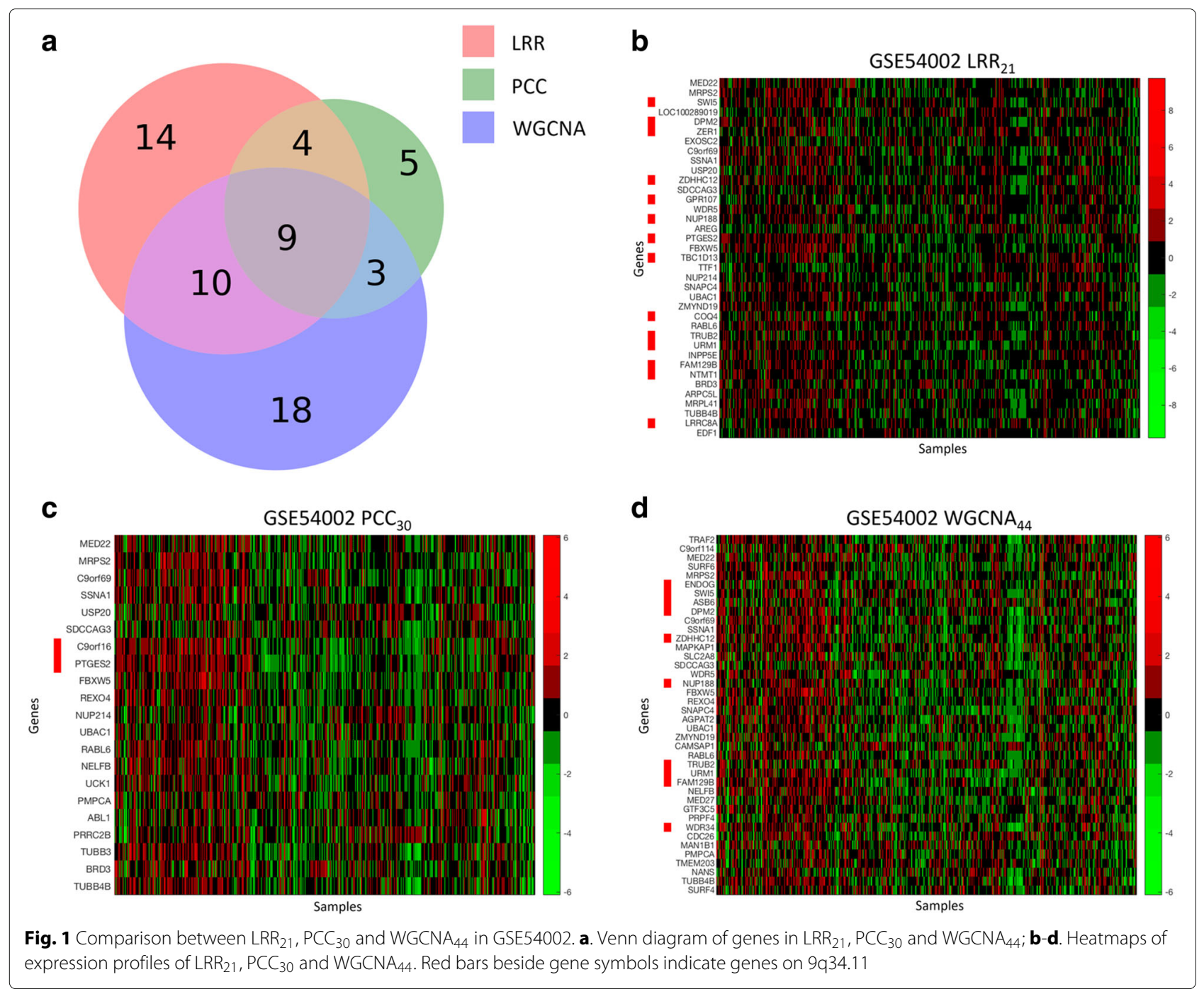

order to show the correlation patterns between genes. We observe that $\mathrm{PCC}_{30}$ and $\mathrm{WGCNA}_{44}$ show a stronger correlation pattern between genes than $\mathrm{LRR}_{21}$, which is confirmed by higher CCI values (Table 3 ). However, some biological annotations are more enriched in $\mathrm{LRR}_{21}$ than in $\mathrm{PCC}_{30}$ or $\mathrm{WGCNA}_{44}$. For example, Table 3 shows chromosome band $9 \mathrm{q} 34.11$ is more enriched in $\mathrm{LRR}_{21}$ with $P$ value $=2.01 \times 10^{-26}$. Figure 2 further shows that genes on the enriched $9 q 34.11$ chromosome band in $\mathrm{LRR}_{21}$

Table 3 Summary of $\mathrm{LRR}_{21}, \mathrm{PCC}_{30}$ and WGCNA 44 in GSE54002

\begin{tabular}{llll}
\hline Module & LRR $_{21}$ & PCC $_{30}$ & WGCNA $_{44}$ \\
\hline number of genes & 37 & 21 & 40 \\
$\mathrm{CCl}$ & 0.385 & 0.486 & 0.420 \\
number of genes on 9q34.11 & 14 & 2 & 10 \\
$\begin{array}{l}P \text { value of enrichment analysis of 9q34.11 } \\
\text { 2.01e-26 }\end{array}$ & $2.38 \mathrm{e}-3$ & $4.57 \mathrm{e}-17$ \\
\hline
\end{tabular}

share similar CNV patterns in the TCGA and METABRIC breast cancer patient cohorts.

The aforementioned results suggest that by using LRR and allowing expression profiles of gene modules to have higher subspace dimensionality, we can identify biological annotations such as chromosome bands that are missed by correlation based GCNA algorithms. This may further lead to new discoveries of cancer-related structural mutations such as CNVs. Figure 3 provides the number of enriched GO BPs and chromosome bands using LRR, PCC based methods and WGCNA with a $0.01 P$ value cutoff. Our method not only produces results with substantial overlap between current GCNA methods in finding enriched biological annotations, but can also discover new related biological annotations. Such advantages give our method the potential to be combined with current GCNA methods to a get better understanding of gene expression data. Tables 4 and 5 list the most significant GO BPs 


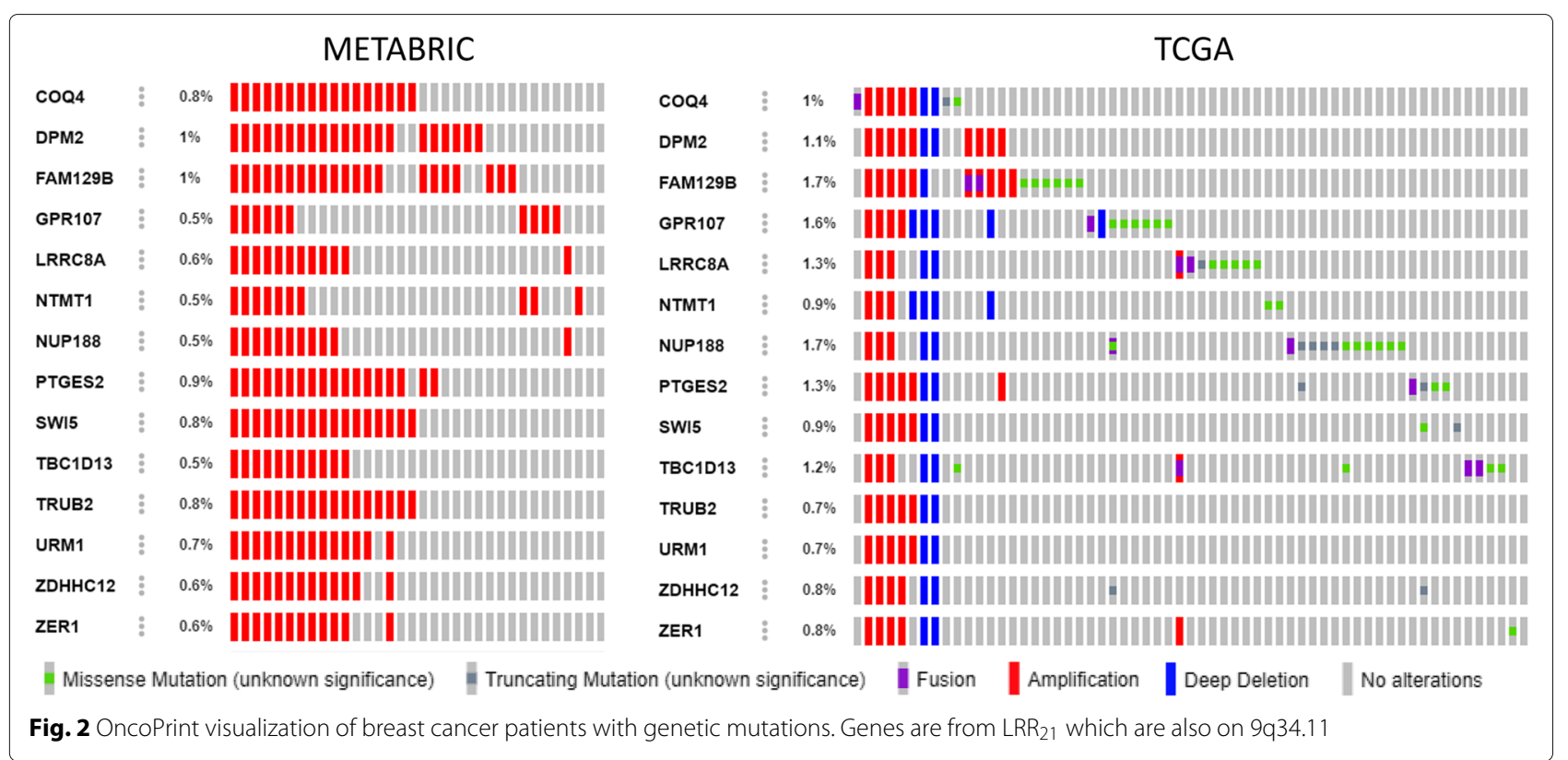

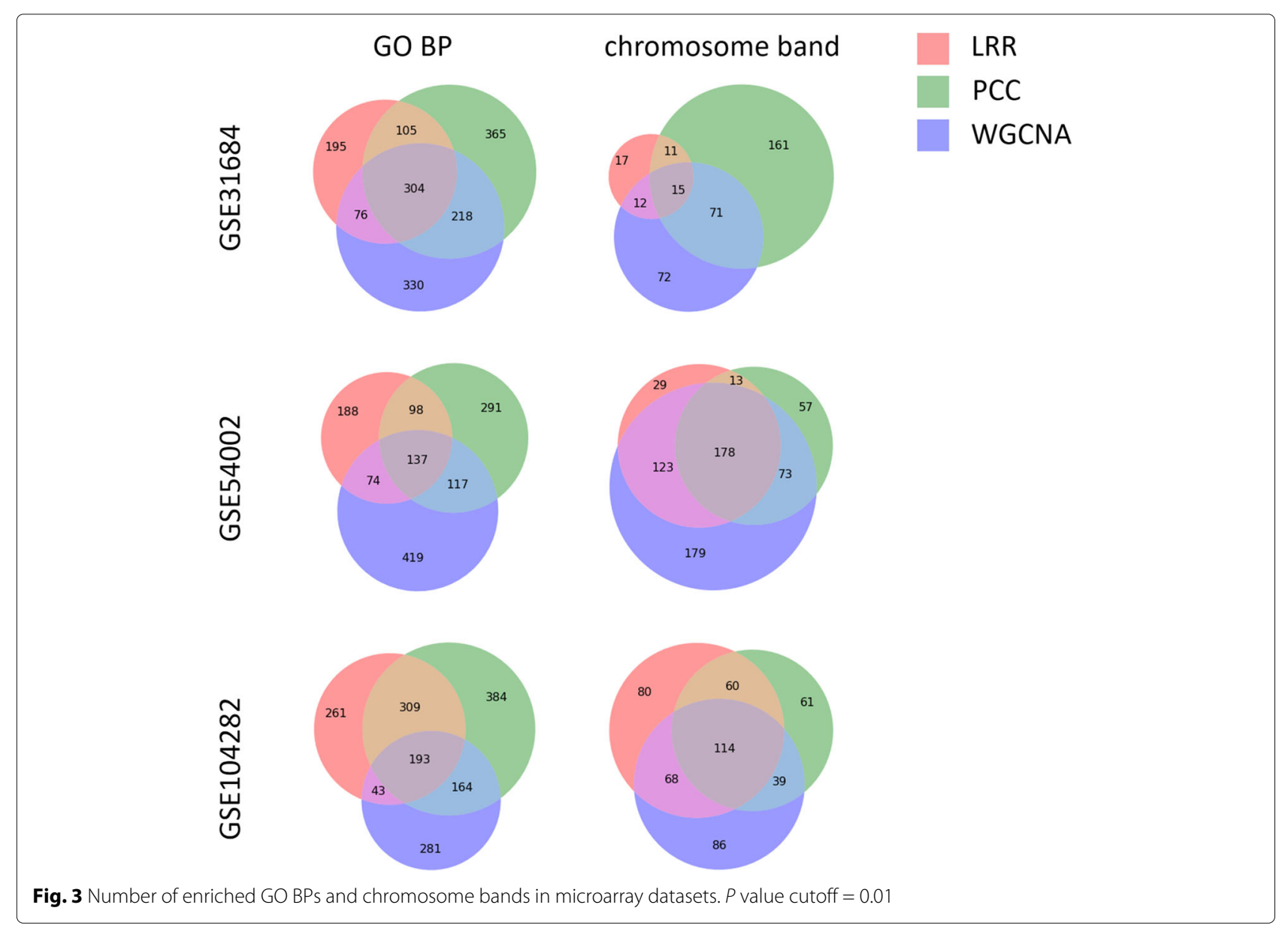


Table 4 The most significant GO BPs only enriched in our method

\begin{tabular}{|c|c|c|c|}
\hline GO ID & Name & $P$ value & Module ID \\
\hline \multicolumn{4}{|l|}{ GSE31684 } \\
\hline GO:0043547 & $\begin{array}{l}\text { positive regulation } \\
\text { of GTPase activity }\end{array}$ & $3.59 \mathrm{e}-06$ & 4 \\
\hline GO:0036336 & $\begin{array}{l}\text { dendritic cell } \\
\text { migration }\end{array}$ & 7.73e-06 & 4 \\
\hline GO:0043087 & $\begin{array}{l}\text { regulation of } \\
\text { GTPase activity }\end{array}$ & $2.89 \mathrm{e}-05$ & 4 \\
\hline GO:0006929 & $\begin{array}{l}\text { substrate- } \\
\text { dependent cell } \\
\text { migration }\end{array}$ & $9.66 \mathrm{e}-05$ & 13 \\
\hline GO:0070232 & $\begin{array}{l}\text { regulation of T cell } \\
\text { apoptotic process }\end{array}$ & $1.07 e-04$ & 4 \\
\hline \multicolumn{4}{|l|}{ GSE54002 } \\
\hline GO:0000082 & $\begin{array}{l}\text { G1/S transition of } \\
\text { mitotic cell cycle }\end{array}$ & $1.27 \mathrm{e}-07$ & 6 \\
\hline GO:0031109 & $\begin{array}{l}\text { microtubule } \\
\text { polymerization or } \\
\text { depolymerization }\end{array}$ & $7.13 e-07$ & 6 \\
\hline \multirow[t]{2}{*}{ GO:0000083 } & $\begin{array}{l}\text { regulation of } \\
\text { transcription } \\
\text { involved in }\end{array}$ & $8.48 \mathrm{e}-07$ & 6 \\
\hline & $\begin{array}{l}\text { G1/S transition of } \\
\text { mitotic cell cycle }\end{array}$ & & \\
\hline GO:0007019 & $\begin{array}{l}\text { microtubule } \\
\text { depolymerization }\end{array}$ & $6.00 \mathrm{e}-06$ & 6 \\
\hline GO:0051783 & $\begin{array}{l}\text { regulation of } \\
\text { nuclear division }\end{array}$ & $7.21 \mathrm{e}-06$ & 6 \\
\hline \multicolumn{4}{|l|}{ GSE102484 } \\
\hline GO:0050766 & $\begin{array}{l}\text { positive regulation } \\
\text { of phagocytosis }\end{array}$ & $1.70 \mathrm{e}-06$ & 1 \\
\hline GO:0006271 & $\begin{array}{l}\text { DNA strand } \\
\text { elongation } \\
\text { involved in DNA } \\
\text { replication }\end{array}$ & $1.95 \mathrm{e}-06$ & 10 \\
\hline GO:0022616 & $\begin{array}{l}\text { DNA strand } \\
\text { elongation }\end{array}$ & $1.95 \mathrm{e}-06$ & 10 \\
\hline GO:0050764 & $\begin{array}{l}\text { regulation of } \\
\text { phagocytosis }\end{array}$ & $3.18 \mathrm{e}-06$ & 1 \\
\hline GO:0070374 & $\begin{array}{l}\text { positive regulation } \\
\text { of ERK1 and ERK2 } \\
\text { cascade }\end{array}$ & $8.79 e-06$ & 1 \\
\hline
\end{tabular}

$P$ value cutoff $=0.01$

and chromosome bands that are only enriched when using our method.

We also observe that for most chromosome bands that are only enriched in our method, genes on these chromosome bands in the corresponding co-expression modules often share similar CNV patterns among patients in the TCGA and METABRIC cohorts.

We also compare overlap of enrichment analysis results between two breast cancer datasets, GSE54002 and GSE102484, using different GCNA methods, which is
Table 5 The most significant chromosome bands only enriched in our method

\begin{tabular}{|c|c|c|c|}
\hline Chromosome band & $P$ value & Module ID & $\begin{array}{l}\text { CNVs validated in separate } \\
\text { cohorts(TCGA, METABRIC) }\end{array}$ \\
\hline \multicolumn{4}{|l|}{ GSE31684 } \\
\hline $4 q 13.3$ & 2.47e-06 & 13 & Yes,NA \\
\hline Yp11.2 & $8.25 e-04$ & 12 & $\mathrm{No}, \mathrm{NA}$ \\
\hline Yq11.223 & $8.25 e-04$ & 12 & $\mathrm{No}, \mathrm{NA}$ \\
\hline $4 q 21.1$ & $8.35 e-04$ & 4 & Yes,NA \\
\hline 19p13.12 & $1.25 e-03$ & 5 & $\mathrm{No}, \mathrm{NA}$ \\
\hline \multicolumn{4}{|l|}{ GSE54002 } \\
\hline $11 \mathrm{p} 15.1$ & $1.92 \mathrm{e}-12$ & 44 & Yes,Yes \\
\hline $11 q 12.2$ & $1.61 \mathrm{e}-06$ & 19 & Yes,Yes \\
\hline $19 q 13.31$ & $1.03 e-05$ & 61 & Yes,Yes \\
\hline $9 q 31.3$ & $2.12 \mathrm{e}-05$ & 26 & No,Yes \\
\hline $9 q 22.33$ & $2.28 \mathrm{e}-05$ & 26 & No,Yes \\
\hline \multicolumn{4}{|l|}{ GSE102484 } \\
\hline 8p11.23 & 3.97e-19 & 53 & Yes,Yes \\
\hline $9 q 34.3$ & $1.36 \mathrm{e}-09$ & 14 & Yes,Yes \\
\hline $15 q 22.31$ & $4.21 e-09$ & 27 & Yes,Yes \\
\hline $13 q 14.3$ & $2.27 e-08$ & 18 & Yes,Yes \\
\hline 7q36.3 & $4.12 \mathrm{e}-08$ & 7 & Yes,Yes \\
\hline
\end{tabular}

$P$ value cutoff $=0.01$

shown in Fig. 4. We observe that LRR and PCC based methods obtain similar fractions of overlap between two datasets, which indicates that our LRR based approach can achieve similar stability with the commonly used PCC based methods. WGCNA produced results with larger overlaps, which may be due to the fact that gene modules detected by WGCNA contains all the genes after pre-processing rather than just densely connected gene modules as in ImQCM. Since information of specific subtypes of breast cancer are unavailable for GSE54002 and GSE102484 on GEO, the difference of the enrichment analysis across these two datasets may come from the difference in breast cancer subtypes.

\section{Difference in $\mathrm{CCl}$ contributes to difference in PPI networks}

To further validate our method, we use PINA2 platform [27] to verify whether LRR could identify gene co-expression modules that form PPI networks with different density. We use the Homo sapiens PPIs database from PINA2 website and map UniProtKB entries to gene symbols through UniProt website (uniprot.org). For a gene co-expression module with $n$ genes and $n_{\text {PPI PPIs }}$ within the module, we define PPI density of the module as $2 n_{\text {PPI }} /(n(n+1))$ since the PINA2 database allows a protein to interact with itself. We found several cases where modules identified by LRR have lower CCI values and higher 


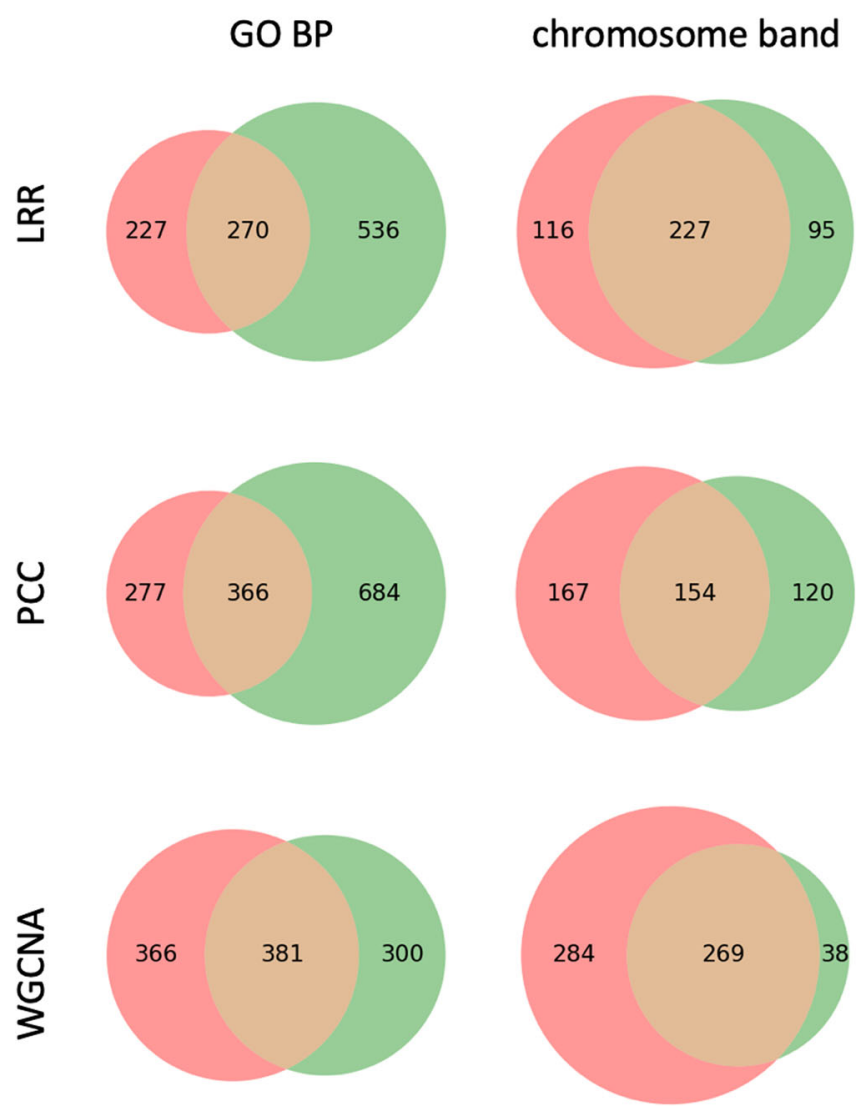

Fig. 4 Number of overlapping enriched GO BPs and chromosome bands. GSE54002 and GSE102484 are two breast cancer datasets

PPI densities than those identified by PCC or WGCNA. Some examples are shown in Table 6 . This suggests that by identifying gene co-expression modules with higher intrinsic dimensionalities, we can find modules that are more densely connected in PPI networks, which indicates that genes in these modules share more similarities in biological functions.

\section{LRR helps identify prognostic gene modules}

To determine whether a gene module has prognostic value, we use a lasso-regularized Cox proportional hazards model to calculate the risk index of each sample based on the expression profiles of the gene module. A leaveone-out cross validation strategy is used to validate our method, where each sample is used as a test sample and classified into a low-risk or a high-risk group. We then use Kaplan-Meier estimator and log-rank test to determine if these two groups have significantly distinct survival. We applied this method on GSE31684, using recurrence free survival months as survival time and recurrence/dod events as censoring information. We removed samples with survival time less than one month from analysis.
Table 6 Examples of modules identified by our method with lower CCls and higher PPI densities

\begin{tabular}{|c|c|c|c|c|}
\hline Dataset & Module(nGenes) & nGenesOverlap & $\mathrm{CCl}$ & PPI density \\
\hline \multirow{2}{*}{ GSE54002 } & $\operatorname{LRR}_{5}(86)$ & \multirow{2}{*}{16} & 0.457 & $3.81 \mathrm{e}-03$ \\
\hline & $\mathrm{PCC}_{14}(28)$ & & 0.498 & 0 \\
\hline \multirow{2}{*}{ GSE54002 } & $\operatorname{LRR}_{5}(86)$ & \multirow{2}{*}{54} & 0.457 & $3.81 \mathrm{e}-03$ \\
\hline & WGCNA $_{18}(94)$ & & 0.467 & $2.38 \mathrm{e}-03$ \\
\hline \multirow{2}{*}{ GSE54002 } & $\operatorname{LRR}_{7}(78)$ & \multirow{2}{*}{18} & 0.340 & $3.12 \mathrm{e}-03$ \\
\hline & $\mathrm{PCC}_{33}(20)$ & & 0.534 & 0 \\
\hline \multirow{2}{*}{ GSE54002 } & $\mathrm{LRR}_{7}(78)$ & \multirow{2}{*}{38} & 0.340 & $3.12 \mathrm{e}-03$ \\
\hline & $W_{G C N A} 26(77)$ & & 0.389 & $1.42 \mathrm{e}-03$ \\
\hline \multirow{2}{*}{ GSE102484 } & $\mathrm{LRR}_{17}(65)$ & \multirow{2}{*}{15} & 0.291 & $2.46 \mathrm{e}-03$ \\
\hline & $\mathrm{PCC}_{24}(28)$ & & 0.400 & 0 \\
\hline \multirow{2}{*}{ GSE102484 } & $\operatorname{LRR}_{17}(65)$ & \multirow{2}{*}{28} & 0.291 & $2.46 \mathrm{e}-03$ \\
\hline & $W_{G C N A}(47)$ & & 0.382 & $1.03 \mathrm{e}-03$ \\
\hline \multirow{2}{*}{ GSE102484 } & $\operatorname{LRR}_{8}(96)$ & \multirow{2}{*}{83} & 0.359 & $5.68 \mathrm{e}-03$ \\
\hline & WGCNA $_{12}(115)$ & & 0.367 & $4.17 \mathrm{e}-03$ \\
\hline
\end{tabular}

nGenes: number of genes in the module nGenes Overlap: number of genes in both modules 


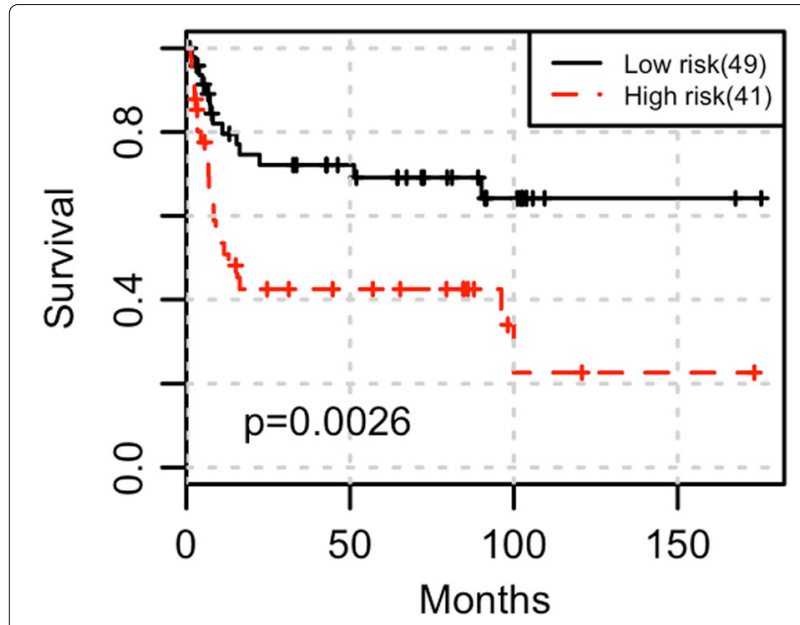

Fig. 5 Kaplan-Meier curve of two groups of samples stratified using gene module LRR 2 in GSE31684. GSE31684 is a bladder cancer dataset

Figure 5 shows that gene co-expression module $\mathrm{LRR}_{2}$, which contains 189 genes, is strongly associated with survival $(P$ value $=0.00260)$. Although gene module $\mathrm{PCC}_{2}$ identified by PCC based method has substantial overlap with $\mathrm{LRR}_{2}\left(\mathrm{PCC}_{2}\right.$ contains 268 genes, where 101 genes are also in $\left.\mathrm{LRR}_{2}\right)$, it is not significantly associated with survival $(P$ value $=0.200)$.

\section{Application to single-cell RNA sequencing data}

Single-cell RNA sequencing (scRNA-seq) techniques have provided powerful tools in studying cellular heterogeneity. In this section, we demonstrate that our method can be extended to scRNA-seq data by applying it on a large scRNA-seq dataset of melanoma cells (GSE72056) [28]. GSE72056 contains 1257 malignant cells, 3256 nonmalignant cells and 132 unresolved cells with expression profiles of 23,686 genes. Only the malignant cells were used for gene co-expression analysis.

Expression level of gene $i$ in cell $j$ is quantified as $G_{i j}=\log _{2}\left(\mathrm{TPM}_{i j} / 10+1\right)$, where $\mathrm{TPM}_{i j}$ is Transcript-PerMillion (TPM) for gene $i$ in cell $j$. In scRNA-seq, dropout event often occurs due to the low number of RNA transcriptomes, which means that expression measurements of some random sampling of transcripts can be missed as zeroes. To account for the dropout events and noise in scRNA-seq data, a different pre-processing method was applied. Firstly, we remove genes with zero expression levels in all cells. Then, we filter out genes with the lowest $80 \%$ of mean expression level or genes with the lowest $80 \%$ of variance. In GSE72056, 3630 genes were retained after pre-processing.

In total, 18, 11 and 16 gene co-expression modules were identified by our method, PCC based ImQCM and WGCNA respectively. Figure 6 provides the number of enriched GO BPs and chromosome bands with a 0.01 $P$ value cutoff. Similar to results in microarray datasets, our method produces results with substantial overlap with existing GCNA methods, while demonstrating the ability to discover new related biological annotations. Table 7 and Table 8 list the most significant GO BPs and chromosome bands that are only enriched when using our method. TCGA skin cutaneous melanoma dataset (363 patients) was used to validate chromosome bands enrichment results. We observe that a large number of patients (3\% - 9\%) in the TCGA cohort have CNVs in genes on the chromosome bands that are uniquely enriched by our method.

Similar to results in microarray datasets, we also observe that difference in CCI values of gene coexpression modules can contributes to difference in enriched biological annotations and density of PPI networks in scRNA-seq data. For example, module $\mathrm{LRR}_{2}$ (112 genes, $\mathrm{CCI}=0.282)$ and module $\mathrm{PCC}_{3}$ (95 genes, $\mathrm{CCI}=0.324)$ have large overlap with 45 genes in common. However, GO:0044237 (cellular metabolic process) is more enriched in $\mathrm{LRR}_{2}$ ( $P$ value $5.85 \mathrm{e}-42$ vs. $2.84 \mathrm{e}-31$ ) and $\mathrm{LRR}_{2}$ has higher PPI density (0.0786 vs. 0.0708).

\section{Discussion}

In this paper, we present a new approach for generalizing the traditional correlation-based GCNA methods beyond

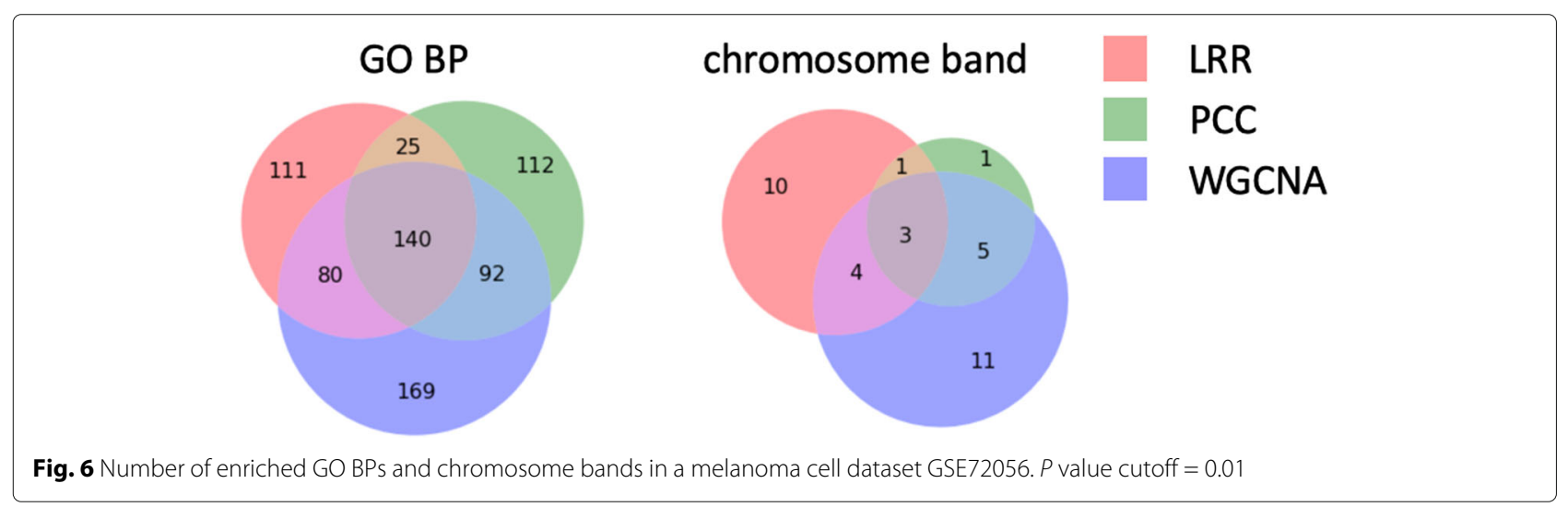


Table 7 The most significant GO BPs only enriched using our method in GSE72056

\begin{tabular}{llll}
\hline GO ID & Name & $P$ value & Module ID \\
\hline GO:0006259 & DNA metabolic process & $1.32 \mathrm{e}-10$ & 6 \\
GO:0006260 & DNA replication & $2.59 \mathrm{e}-07$ & 6 \\
GO:0006281 & DNA repair & $3.17 \mathrm{e}-06$ & 6 \\
GO:0006261 & DNA-dependent DNA replication & $5.10 \mathrm{e}-06$ & 6 \\
GO:0046794 & transport of virus & $8.60 \mathrm{e}-06$ & 2 \\
\hline
\end{tabular}

$P$ value cutoff $=0.01$

pairwise relationships between genes. This generalized GCNA method greatly expands the scope for exploring complex relationships among genes. This approach combines a subspace clustering algorithm, LRR, with a weighted network mining algorithm, $1 \mathrm{mQCM}$, and makes two major improvements comparing with previous methods. Firstly, comparing with current GCNA algorithms, which mostly calculates the similarity between genes based on pairwise correlation coefficients, we introduce subspace clustering into GCNA to find new biologically meaningful gene modules that can not be characterized based on pairwise relationships. Since the LRR approach does not limit the dimensionality of the subspace, it can accommodate complex relationships which often imply multiple gene or pathway interactions or more variable structural variations. Secondly, comparing with current subspace clustering algorithms in $[17,18]$, which assign every gene into a cluster, we choose to use ImQCM, which identifies densely connected modules such as quasicliques in weighted networks. Our method is more consistent with the fact that some genes could participate in multiple biological processes. In addition, our method can be applied to different kinds of gene expression data, including microarray data and scRNA-seq data.

Despite the advantages demonstrated in this paper, there still exists limitations in our method. As mentioned in [29], when using the data itself as the dictionary, LRR may not be able to exactly recover the subspaces when data contains certain types of errors, such as dense noise. Moreover, a critical issue of LRR is how to estimate or

Table 8 The most significant chromosome bands only enriched using our method in GSE72056

\begin{tabular}{lll}
\hline chromosome band & $P$ value & Module ID \\
\hline $5 p 15.33$ & $1.41 \mathrm{e}-06$ & 18 \\
$6 \mathrm{p} 22.1$ & $3.44 \mathrm{e}-05$ & 15 \\
$17 \mathrm{q} 11.2$ & $8.46 \mathrm{e}-05$ & 11 \\
$17 \mathrm{q} 11-\mathrm{q} 12$ & $1.83 \mathrm{e}-03$ & 11 \\
$6 \mathrm{q} 24.1-\mathrm{q} 24.2$ & $1.83 \mathrm{e}-03$ & 11 \\
\hline
\end{tabular}

$P$ value cutoff $=0.01$ select the parameter $\lambda$. When data is contaminated by various errors, the selection of $\lambda$ could be quite challenging. We used a cross-validation style approach to overcome this challenge. However, our approach is quite computationally expensive and efficient ways for parameter estimation should be studied in the future.

\section{Conclusion}

In conclusion, we developed a new generalized gene coexpression analysis algorithm based on subspace clustering that works beyond pairwise relationships between genes. Correlations between genes have been shown to be very useful in identifying gene co-expression modules with biological meanings. However, our method provides a complement to existing GCNA methods by using subspace clustering to identify gene co-expression modules with expression profiles of higher intrinsic dimensionalities. We demonstrate that our method can be applied to various types of gene expression data, including microarray data and the emerging scRNA-seq data. By combining our method with other GCNA tools, we can obtain a more comprehensive understanding of gene expression data.

\section{Abbreviations}

ALM: Augmented lagrange multiplier; CCl: Centralized concordance index; CNA: Copy number alternation; CNV: Copy number variation; GCNA: Gene co-expression network analysis; GEO: Gene expression omnibus; GO BP: Gene ontology biological process; ImQCM: Local maximal quasi-clique merger; LRR: Low-rank representation; PCC: Pearson correlation coefficient; PPI:

Protein-protein interaction; SCC: Spearman correlation coefficient; scRNA-seq: Single-cell RNA sequencing; SSC: Sparse subspace clustering; TCGA: The cancer genome atlas; TPM: Transcript-per-million

\section{Acknowledgements}

Not applicable.

\section{Funding}

This work is partially supported by IUSM startup fund, the NCI ITCR U01 (CA188547) and Indiana University Precision Health Initiative. Publication costs are funded by Indiana University Precision Health Initiative.

\section{Availability of data and materials}

All the datasets can be accessed through Gene Expression Omnibus. The code is available at https://github.com/txWang/generalized_GCNA.

\section{About this supplement}

This article has been published as part of BMC Bioinformatics Volume 20 Supplement 7, 2019: Selected papers from the 12th International Conference on Computational Systems Biology (ISB 2018). The full contents of the supplement are available online at https://bmcbioinformatics.biomedcentral.com/articles/ supplements/volume-20-supplement-7.

\section{Authors' contributions}

Conceived and designed the experiments: TW, KH. Performed the experiments and analyzed the data: TW, JZ. Developed the structure and arguments for the paper: TW, JZ, KH. Drafted the manuscript: TW, KH. Edited the manuscript: JZ, $\mathrm{KH}$. All the authors reviewed and approved of the final manuscript.

Ethics approval and consent to participate

Not applicable.

Consent for publication

Not applicable. 


\section{Competing interests}

The authors declare that they have no competing interests.

\section{Publisher's Note}

Springer Nature remains neutral with regard to jurisdictional claims in published maps and institutional affiliations.

\section{Author details}

${ }^{1}$ Department of Computer Science, Indiana University Bloomington, 47408 Bloomington, IN, USA. ${ }^{2}$ Department of Medical and Molecular Genetics, Indiana University School of Medicine, 46202 Indianapolis, IN, USA

${ }^{3}$ Department of Medicine, Indiana University School of Medicine, 46202 Indianapolis, IN, USA. ${ }^{4}$ Regenstrief Institute, 46202 Indianapolis, IN, USA.

\section{Published: 1 May 2019}

\section{References}

1. Pujana MA, Han J-DJ, Starita LM, Stevens KN, Tewari M, Ahn JS, Rennert G, Moreno V, Kirchhoff T, Gold B, et al. Network modeling links breast cancer susceptibility and centrosome dysfunction. Nat Genet. 2007;39(11):1338.

2. Kais Z, Barsky SH, Mathsyaraja H, Zha A, Ransburgh DJ, He G, Pilarski RT, Shapiro CL, Huang K, Parvin JD. KIAA0101 interacts with BRCA1 and regulates centrosome number. Mol Cancer Res. 2011;9(8):1091-1099.

3. Zhang J, Lu K, Xiang Y, Islam M, Kotian S, Kais Z, Lee C, Arora M, Liu H-w, Parvin JD, et al. Weighted frequent gene co-expression network mining to identify genes involved in genome stability. PLoS Comput Biol. 2012;8(8):1002656.

4. MacLennan NK, Dong J, Aten JE, Horvath S, Rahib L, Ornelas L, Dipple KM McCabe ER. Weighted gene co-expression network analysis identifies biomarkers in glycerol kinase deficient mice. Mol Genet Metab. 2009;98(1):203-14.

5. Zhang J, Xiang Y, Ding L, Borlawsky TB, Ozer HG, Jin R, Payne P, Huang K et al. Using gene co-expression network analysis to predict biomarkers for chronic lymphocytic leukemia. BMC Bioinformatics. 2010;11 (Suppl 9):5.

6. Zhao J, Hu X, He T, Li P, Zhang M, Shen X. An edge-based protein complex identification algorithm with gene co-expression data (pcia-geco). IEEE Trans Nanobioscience. 2014;13(2):80-88.

7. Zhang J, Ni S, Xiang Y, Parvin JD, Yang Y, Zhou Y, Huang K. Gene co-expression analysis predicts genetic aberration loci associated with colon cancer metastasis. Int J Comput Biol Drug Des. 2013;6(1-2):60-71.

8. Xu Y, DuanMu H, Chang Z, Zhang S, Li Z, Li Z, Liu Y, Li K, Qiu F, LiX. The application of gene co-expression network reconstruction based on cnvs and gene expression microarray data in breast cancer. Mol Biol Rep. 2012;39(2):1627-37.

9. Hu H, Yan X, Huang Y, Han J, Zhou XJ. Mining coherent dense subgraphs across massive biological networks for functional discovery. Bioinformatics. 2005;21(12(Suppl 1)):213-21.

10. Langfelder P, Horvath S. Wgcna: an r package for weighted correlation network analysis. BMC Bioinformatics. 2008;9(1):559.

11. Li A, Horvath S. Network module detection: Affinity search technique with the multi-node topological overlap measure. BMC Res Notes. 2009;2:142.

12. Xiang $Y$, Zhang C-Q, Huang K. Predicting glioblastoma prognosis networks using weighted gene co-expression network analysis on tcga data. BMC Bioinformatics. 2012;13(Suppl 2):12.

13. Zhang J, Huang K. Normalized imqcm: An algorithm for detecting weak quasi-cliques in weighted graph with applications in gene co-expression module discovery in cancers. Cancer Inf. 2014;13s3:14021.

14. Vidal R. Subspace clustering. IEEE Signal Proc Mag. 2011;28(2):52-68.

15. Hong W, Wright J, Huang K, Ma Y. Multiscale hybrid linear models for lossy image representation. IEEE Trans Image Proc. 2006;15(12):3655-71.

16. Yang AY, Wright J, Ma Y, Sastry SS. Unsupervised segmentation of natural images via lossy data compression. Computer Vision and Image Understanding. 2008;110(2):212-225.

17. Elhamifar E, Vidal R. Sparse subspace clustering: Algorithm, theory, and applications. IEEE Trans Pattern Anal Mach Intell. 2013;35(11):2765-81.

18. Liu G, Lin Z, Yu Y. Robust subspace segmentation by low-rank representation. In: Proceedings of the 27 th International Conference on Machine Learning; 2010. p. 663-670.

19. Cheng Y, Church GM. Biclustering of expression data. Proc Int Conf Intell Syst Mol Biol. AAAI. 2000;8:93-103. https://www.ncbi.nlm.nih.gov/ pubmed/10977070.
20. Getz G, Levine E, Domany E. Coupled two-way clustering analysis of gene microarray data. Proc Natl Acad Sci. 2000;97(22):12079-84.

21. Lazzeroni $L$, Owen A. Plaid models for gene expression data. Stat Sin. 2002:61-86. https://www.jstor.org/stable/2430703.

22. Elhamifar E, Vidal R. Sparse subspace clustering. In: 2009 IEEE Conference on Computer Vision and Pattern Recognition. IEEE; 2009. p. 2790-2797.

23. Jiang D, Tang C, Zhang A. Cluster analysis for gene expression data: a survey. IEEE Trans Knowl Data Eng. 2004;16(11):1370-86.

24. Gao J, Aksoy BA, Dogrusoz U, Dresdner G, Gross B, Sumer SO, Sun Y, Jacobsen A, Sinha R, Larsson E, et al. Integrative analysis of complex cancer genomics and clinical profiles using the cbioportal. Sci Signal. 2013;6(269):1-1.

25. Curtis C, Shah SP, Chin S-F, Turashvili G, Rueda OM, Dunning MJ, Speed D, Lynch AG, Samarajiwa S, Yuan Y, et al. The genomic and transcriptomic architecture of 2,000 breast tumours reveals novel subgroups. Nature. 2012;486(7403):346.

26. Han Z, Zhang J, Sun G, Liu G, Huang K. A matrix rank based concordance index for evaluating and detecting conditional specific co-expressed gene modules. BMC genomics. 2016;177(Suppl 7):519.

27. Cowley MJ, Pinese M, Kassahn KS, Waddell N, Pearson JV, Grimmond SM, Biankin AV, Hautaniemi S, Wu J. Pina v2 0: mining interactome modules. Nucleic Acids Res. 2011;40(D1):862-5

28. Tirosh I, Izar B, Prakadan SM, Wadsworth MH, Treacy D, Trombetta JJ, Rotem A, Rodman C, Lian C, Murphy G, et al. Dissecting the multicellular ecosystem of metastatic melanoma by single-cell rna-seq. Science. 2016;352(6282):189-96.

29. Liu G, Lin Z, Yan S, Sun J, Yu Y, Ma Y. Robust recovery of subspace structures by low-rank representation. IEEE Trans Pattern Anal Mach Intell. 2013;35(1):171-84

\section{Ready to submit your research? Choose BMC and benefit from}

- fast, convenient online submission

- thorough peer review by experienced researchers in your field

- rapid publication on acceptance

- support for research data, including large and complex data types

- gold Open Access which fosters wider collaboration and increased citations

- maximum visibility for your research: over $100 \mathrm{M}$ website views per year

At BMC, research is always in progress.

Learn more biomedcentral.com/submissions 